Rev. Int. Contam. Ambie. 32 (4) 375-384, 2016

DOI: 10.20937/RICA.2016.32.04.01

\title{
EVALUACIÓN DE ELEMENTOS TRAZA EN AGUA DE RÍO Y MANANTIAL DEL ÁREA MINERA DE OCAMPO, CHIHUAHUA, MÉXICO
}

\author{
Karla Ozuky CHACÓN CHUMACERO, Carmelo PINEDO ÁLVAREZ y \\ Marusia RENTERÍA VILLALOBOS*
}

Departamento de Recursos Naturales, Facultad de Zootecnia y Ecología, Universidad Autónoma de Chihuahua, Avenida Universidad y Pascual Orozco s/n, Universidad, Chihuahua, Chihuahua, México, C.P. 31110

*Autor para correspondencia: marusia@us.es

(Recibido mayo 2015; aceptado febrero 2016)

Palabras clave: metales pesados, zona impactada

\section{RESUMEN}

La industria forestal y la actividad minera contribuyen con más del $80 \%$ en la economía de la ecorregión templada de estado de Chihuahua, México. El objetivo de este trabajo fue evaluar las concentraciones de algunos elementos traza en muestras de agua de ríos y manantiales (que son las principales fuentes de suministro para uso y consumo humano) en el ambiente circundante del corredor minero de oro y plata en el distrito de Ocampo, localizado en el centro oeste del estado. Los parámetros de temperatura, sólidos disueltos totales, turbidez y $\mathrm{pH}$ fueron medidos in situ. Las concentraciones de elementos mayoritarios ( $\mathrm{Ca}$ y $\mathrm{Mg}$ ) y traza $(\mathrm{Li}, \mathrm{Ni}, \mathrm{Sb}, \mathrm{Se}, \mathrm{Cu}$ y $\mathrm{Zn}$ ) fueron obtenidas con un espectrómetro de emisión óptica. El promedio de la concentración de Ca fue más alto en agua de río en comparación con el de manantial, mientras que los contenidos de $\mathrm{Mg}$ sólo fueron detectados en agua de río. Los elementos traza medidos en agua de río y manantial presentaron una tendencia decreciente según contenidos de $\mathrm{Ni}>\mathrm{Cu}>\mathrm{Zn}>\mathrm{Li}>\mathrm{Se}>\mathrm{Sb}$. Las concentraciones más altas de elementos traza fueron encontradas en un área altamente impactada por actividades relacionadas con la minería. La mayoría de las concentraciones de elementos traza estuvieron por debajo de los límites permisibles establecidos por la Agencia de Protección Ambiental de Estados Unidos de América y la normatividad mexicana. Sin embargo, las concentraciones de $\mathrm{Cu}$, Ni y $\mathrm{Zn}$ obtenidas en agua de río fueron más altas que las marcadas por las directrices para la vida acuática establecidas en Canadá. Aunque, la concentración elemental determinada está por debajo de los límites permisibles, estos estudios son de gran importancia porque el agua de esta región es usada para consumo humano, como agua de riego y para la industria.

Key words: heavy metal, affected area

\begin{abstract}
The forestry industry and gold mining contribute to more than $80 \%$ of the economy of the temperate ecoregion of Chihuahua, Mexico. The aim of this work was to assess the concentrations of some trace elements in water samples from the rivers and the springs in the surrounding environment of the gold-silver runner mining in the
\end{abstract}


Ocampo district. It is located in the center-west of Chihuahua, Mexico. Water sampling was performed on rivers and springs, which are the main water supply sources for human consumption. Temperature, total dissolved solids, turbidity and $\mathrm{pH}$ parameters were measured in situ. Some mayor (Ca and $\mathrm{Mg}$ ) and trace elements ( $\mathrm{Li}, \mathrm{Ni}, \mathrm{Sb}, \mathrm{Se}$, $\mathrm{Cu}$, and $\mathrm{Zn}$ ) were quantified by inductively coupled plasma of optical emission. The average of $\mathrm{Ca}$ concentration in water was higher in rivers than in springs, whereas $\mathrm{Mg}$ contents were only detected in river water. Trace elements measured in waters of river and spring were in decrease trend $\mathrm{Ni}>\mathrm{Cu}>\mathrm{Zn}>\mathrm{Li}>\mathrm{Se}>\mathrm{Sb}$. The highest trace element concentrations were found in an area highly impacted by activities related to the mining. Most of trace elements concentrations assessed in this work were lower than limits for drinking water marked by Unites States Environmental Protection Agency and Mexican regulations. However, concentrations of $\mathrm{Cu}, \mathrm{Ni}$, and $\mathrm{Zn}$ obtained in water from rivers are higher than Canadian guidelines for protection of the aquatic life. Although, elemental concentrations determined here are below of the allowed limits, these studies are important because the water in this region have different uses such as human consumption, water supply to agricultural lands, and to industry.

\section{INTRODUCCIÓN}

La industria minera es una de las actividades que tiene una contribución importante en el crecimiento económico de México. En particular, esta actividad aunada al aprovechamiento forestal, contribuye con más del $80 \%$ en la economía de la ecorregión templada del estado de Chihuahua. En general, tiene impactos políticos, económicos, sociales y ambientales a escalas global y local (Fernández-Manso et al. 2012). Además, en cuanto a la estructura del paisaje forestal tiene efectos significativos en las comunidades locales y en el ambiente (Younger 1997).

La estrecha relación entre la forma de vida y la explotación de los recursos naturales causa una grave contaminación ambiental, pérdida de recursos bióticos y abióticos, fragmentación de hábitat, erosión y contaminación por metales pesados (Hilson y Van Der Vorst 2002, Bridge 2004, Kumah 2006). La contaminación del agua es un gran problema que se relaciona con el crecimiento económico de cualquier país y región (Srinivasa y Govil 2008). El agua de manantiales y los flujos superficiales son la principal fuente para uso doméstico en áreas rurales (Ansari et al. 1999). Su calidad es de vital importancia debido a su uso en las actividades antrópicas (Torres et al. 2009).

La contaminación por metales potencialmente nocivos en sedimentos, suelo y agua es una de las amenazas más importantes para la calidad del ambiente y de la salud humana (Gómez-Álvarez et al. 2011). El agua se altera en sus propiedades biológicas, físicas y químicas, debido a varios agentes de la naturaleza y por la forma en que se utiliza (Metcalf y Eddy 1996).
Las actividades mineras están asociadas con los impactos ambientales, incluyendo la contaminación por metales de los sedimentos y de los sistemas acuáticos (Hun-Bok et al. 2005). La minería está asociada con la modificación de la superficie, lo cual afecta la cuenca y el régimen hidrológico tanto en cantidad como en calidad (Bud et al. 2007). Ejemplo de lo anterior son los metales pesados que ingresan al ambiente acuático principalmente por descargas directas de fuentes industriales (Alloway y Ayres 1993). Específicamente, los problemas ambientales relacionados con los depósitos de mineral son el drenaje ácido y las altas concentraciones de metales pesados en el agua de drenaje de los yacimientos mineros (Carrillo-Chávez et al. 2003).

El distrito de Ocampo es uno de los corredores mineros de gran importancia en actividades económicas del estado de Chihuahua. Su localización es al centro oeste del estado, el cual contempla el parque nacional de Basaseachi y el área de protección de flora y fauna Tutuaca. Los ecosistemas forestales ubicados en este distrito evidencian procesos de degradación y contaminación ambiental producidos por las actividades forestales y por la explotación minera. Además, en esta región nacen los ríos Balloreca, Concheño y Apituchi, los cuales pertenecen a la región hidrológica sur de Sonora (RH09) y drenan agua y sedimentos a las áreas de agricultura de la costa del Pacífico (Villanueva et al. 2009). La geología de la zona se encuentra formada por rocas ígneas extrusivas $(85.4 \%$ riolita-toba ácida) y sedimentarias $(12 \%$ arenisca-conglomerado) y en menor escala por rocas volcánicas del tipo riolíticas terciarias del periodo Cuaternario (CONANP 2014). La composición del suelo es de origen residual y aluvial dominado por 
Feozem, Regosol y Litosol. Esta área se encuentra en un intervalo de altitud de 458 a $2911 \mathrm{msnm}$ y presenta un clima templado subhúmedo con lluvias de verano. El intervalo de precipitación anual en el municipio de Moris es de 600 a 1000 mm (INEGI 2005a), mientras que para el resto de la zona es de 1000 a 1200 mm (INEGI 2005b). Dada la variabilidad fisiográfica de esta región, se encuentran en ella diversos tipos de asociaciones vegetales como el bosque de pino encino y otras coníferas latifoliadas. Ejemplo de lo anterior son: los pinos (Pinus arizonica, Pinus durangensis, Pinus engelmannii, Pinus ayacahuite y Pinus leiophylla), los encinos (Quercus rugosa, Quercus crassifolia, Quercus sideroxyla y Quercus fulva), el táscate (Juniperus deppeana) y el madroño (Arbutus arizonica y Arbutus xalapensis; Azarcoya 2012). De igual manera, entre las nueve principales especies nativas de ictiofauna presentes en los cauces de la región se incluyen: el rodapiedras mexicano (Compostoma ornatum), y otras especies como Catostomus bernardini, Gila nigrescens, Gila robusta, Ictalarus melas, Ictalarus pricei, Lepomis cynaellus y Salmo sp.

Actualmente no existen estudios que permitan conocer el impacto de las actividades mineras sobre la calidad del agua. Por lo anterior, el objetivo de este trabajo fue evaluar las concentraciones de algunos elementos traza en muestras de agua de ríos y manantiales en el ambiente circundante del corredor minero de oro y plata en el distrito de Ocampo, Chihuahua. Con ello se podrá tener un análisis inicial del impacto por contaminación química hacia estos cuerpos de agua que son las principales fuentes de suministro para uso y consumo humano en dicha región.

\section{MATERIALES Y MÉTODOS}

El muestreo se llevó a cabo después de la temporada de lluvias, en el mes de octubre de 2014. Las muestras de agua fueron colectadas de manantiales y ríos que son las principales fuentes de abastecimiento de agua para consumo humano. Los ríos Balloreca, Concheño y Apituchi son permanentes y fueron muestreados en varios puntos a lo largo del flujo de agua. Asimismo, se colectaron muestras de agua de siete manantiales (Fig. 1). Los parámetros de temperatura, sólidos disueltos totales, conductividad eléctrica, turbidez y $\mathrm{pH}$ fueron medidos in situ, con un potenciómetro multiparamétrico (Hanna, modelo HI-98130). La georreferenciación de cada punto de muestreo fue realizada con un sistema de geoposicionamiento global GPS (Garmin, modelo GPSmap 62s).
Cada muestra fue colectada en recipientes de polietileno de $1 \mathrm{~L}$ de capacidad, las cuales fueron preservadas adicionando $\mathrm{HNO}_{3}($ hasta $\mathrm{pH}=2$ ) para ser analizadas en el laboratorio posteriormente. El pretratamiento de los recipientes de muestreo fue realizado como lo indica la norma NOM-001-ECOL -1996 (SEMARNAT 1996).

En el laboratorio, las muestras fueron digeridas en microondas (Anton Paar, modelo Multiwave GO) utilizando $\mathrm{HCl}$ y $\mathrm{HNO}_{3}$ (EPA 2007). La caracterización elemental se realizó mediante un espectrómetro ICP-OES, manufacturado por Perkin Elmer, modelo 8300. Las mediciones realizadas para cada muestra se realizaron por triplicado. Además, se midió una muestra blanco utilizando agua desionizada grado reactivo (Sumilab). Asimismo, para la calibración del equipo se utilizaron soluciones patrón de concentraciones de 1,5 y $10 \mathrm{mg} / \mathrm{L}$ de un estándar de 21 elementos, de alta pureza (STD-As QC 21 elements, $100 \mathrm{mg} / \mathrm{L}$ de cada elemento), marca Perkin Elmer. Los elementos mayoritarios analizados fueron $\mathrm{Ca}$ y $\mathrm{Mg}$, mientras que los elementos traza fueron $\mathrm{As}, \mathrm{Be}$, $\mathrm{Cd}, \mathrm{Cr}, \mathrm{Cu}, \mathrm{Li}, \mathrm{Ni}, \mathrm{Pb}, \mathrm{Sb}, \mathrm{Se}, \mathrm{Sr}$ y Zn. El límite de detección del equipo (LD) para los elementos analizados es del orden de ppb $(\mu \mathrm{g} / \mathrm{L})$ : As $(1)$, Be (0.08), $\mathrm{Ca}$ (0.58), Cd (0.05), Cr (0.15), Cu (0.69), Li (0.23), $\mathrm{Mg}$ (0.3), Ni (0.35), Pb (1.1), Sb (1.1), Se (1.3), Sr (0.01), Zn (0.88). A partir de los elementos mayoritarios se pudo calcular la dureza. Generalmente, la dureza se expresa en ppm de $\mathrm{CaCO}_{3}$ o meq/L, por lo que se determina utilizando la ecuación 1 :

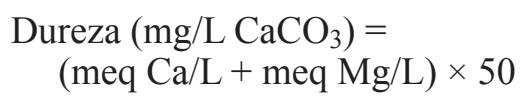

Donde, un meq/L $\mathrm{CaCO} 3$ equivale a $50 \mathrm{ppm}$ $\mathrm{CaCO} 3$.

\section{RESULTADOS}

En el cuadro I se muestran los datos de temperatura $(\mathrm{T}), \mathrm{pH}$, sólidos disueltos totales (SDT), conductividad eléctrica (CE), turbidez (TB) y las coordenadas geográficas de cada sitio de muestreo. La T del agua de manantial fue ligeramente más alta que la de las muestras de río, con valores promedio de 18.3 y 16.1 en agua de río y manantial, respectivamente. Los valores de $\mathrm{pH}$ en agua de río fueron de neutral a ligeramente básico, mientras que en agua de manantial el $\mathrm{pH}$ fue de ligeramente ácido a neutro. Los valores de los SDT determinados en muestras de río y manantial se encontraron en un 


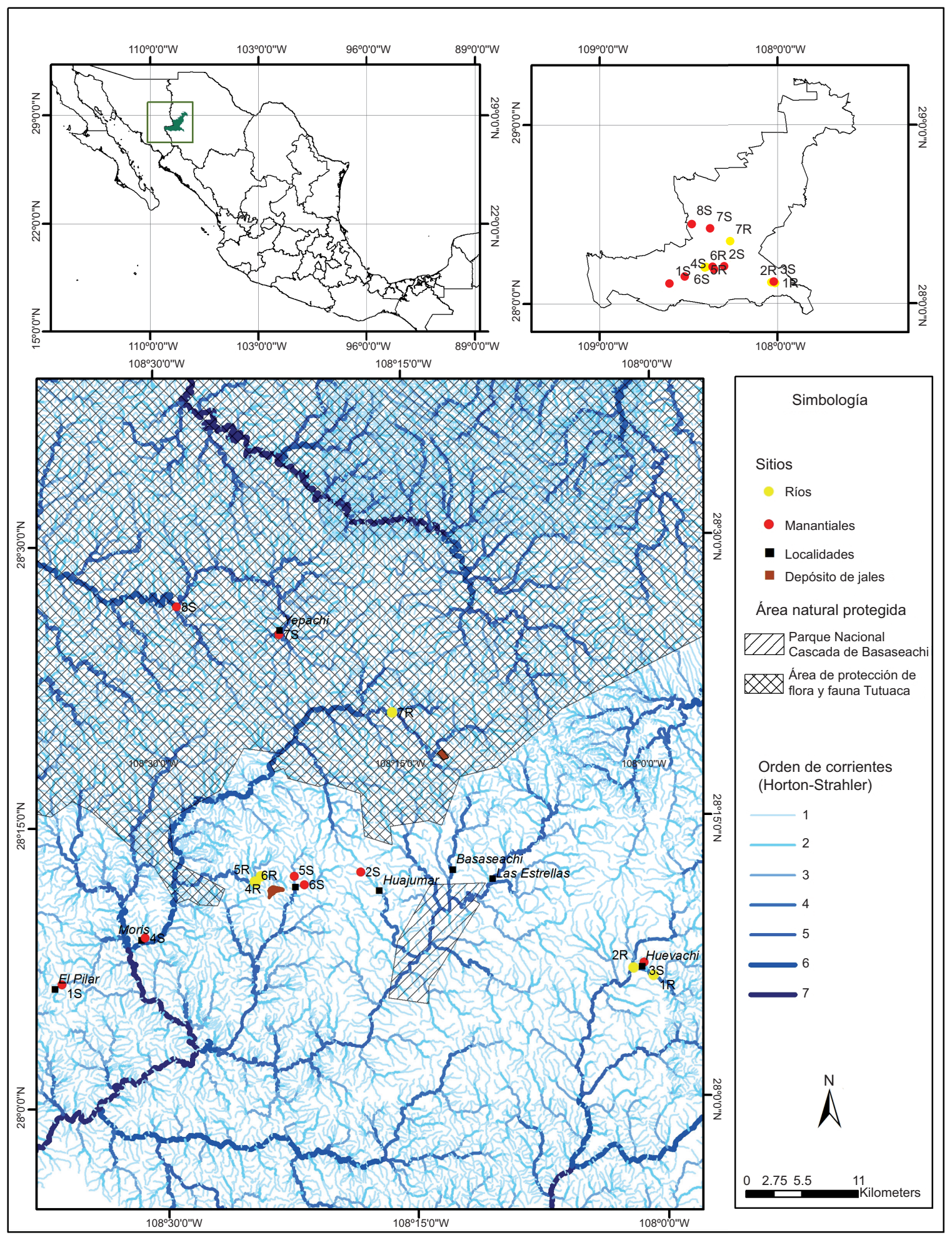

Fig. 1. Localización del área de estudio con los puntos de muestreo de agua de río (R) y manantial (S)

intervalo de concentraciones de 23 a $334 \mathrm{mg} / \mathrm{L}$ y de 13 a $242 \mathrm{mg} / \mathrm{L}$, respectivamente. Los valores de $\mathrm{pH}$ y SDT se encuentran dentro de los límites permisibles propuestos en las diferentes agencias de regulación para agua de consumo humano (EPA 2000, SSA 1994, WHO 2008). 
CUADRO I. RESULTADOS DE LOS PARÁMETROS MEDIDOS IN SITU DE LAS MUESTRAS DE LOS RÍOS (BALLORECA, CONCHEÑO Y APITUCHI) Y MANANTIALES, ASÍ COMO LOS LÍMITES PERMISIBLES PARA CADA UNO DE ELLOS PROPUESTOS POR LAS DIFERENTES AGENCIAS REGULADORAS DE CALIDAD DE AGUA

\begin{tabular}{lcccccccc}
\hline Muestra & $\begin{array}{c}\mathrm{T} \\
\left({ }^{\circ} \mathrm{C}\right)\end{array}$ & $\mathrm{pH}$ & $\begin{array}{c}\mathrm{SDT} \\
(\mathrm{mg} / \mathrm{L})\end{array}$ & $\begin{array}{c}\mathrm{TB} \\
(\mathrm{UNT})\end{array}$ & $\begin{array}{c}\mathrm{CE} \\
(\mathrm{mS} / \mathrm{cm})\end{array}$ & $\begin{array}{c}\text { Oeste } \\
\text { X }\end{array}$ & $\begin{array}{c}\text { Norte } \\
\mathrm{Y}\end{array}$ & $\begin{array}{c}\text { Altitud } \\
(\mathrm{msnm})\end{array}$ \\
\hline NORM $^{1}$ & & $6.5-8.5$ & 1000 & 5 & & & & \\
WHO $^{2}$ & & 8.5 & 500 & 0.1 & & & & \\
EPA $^{3}$ & & $6.05-8.05$ & 500 & & & & & \\
FAO $^{4}$ & & 8.5 & 2000 & & 3 & & & \\
Río $(\mathrm{R})$ & & & & & & & & \\
1R & 16.6 & 8.05 & 307 & 1.38 & 6.01 & 793774 & 3112733 & 2118 \\
2R & 20.1 & 7.84 & 334 & 0.13 & 6.6 & 791810 & 3113467 & 2078 \\
3R & 12.5 & 8.09 & 91 & 0.69 & 1.82 & 755063 & 3122482 & 1737 \\
4R & 13.6 & 8.07 & 74 & 1.15 & 1.47 & 755067 & 3122499 & 1739 \\
5R & 15 & 7.97 & 78 & 5.67 & 1.49 & 754474 & 3121946 & 1700 \\
6R & 17.4 & 8.05 & 85 & nd & 1.71 & 754550 & 3121877 & 1700 \\
7R & 17.5 & 8.13 & 23 & 3.05 & 0.48 & 768038 & 3138656 & 1743 \\
\hline & & & & Manantial (S) & & & & \\
\hline 1S & 20.5 & 7.01 & 35 & 17.75 & 0.69 & 735429 & 3111770 & 1423 \\
2S & 15 & 6.87 & 34 & nd & 0.75 & 764897 & 3122924 & 2263 \\
3S & 17.6 & 7.16 & 242 & 0.66 & 4.88 & 792819 & 3113986 & 2120 \\
4S & 25.6 & 7.07 & 35 & 0.87 & 0.71 & 743630 & 3116349 & 770 \\
5S & 16.1 & 7.39 & 35 & 0.87 & 0.71 & 758324 & 3122513 & 1812 \\
6S & 15.4 & 7.61 & 24 & 0.03 & 0.50 & 759324 & 3121674 & 1842 \\
7S & 17.3 & 6.88 & 13 & 1.98 & 0.30 & 756811 & 3146325 & 1780 \\
8S & 19.2 & 7.55 & 13 & 2.76 & 0.71 & 746715 & 3149098 & 1486 \\
\hline
\end{tabular}

${ }^{1}$ NOM-127-SSA1-1994, (Secretaría de Salud 1994), agua de consumo humano.

${ }^{2}$ Organización Mundial de la Salud, (WHO 2008), agua de consumo humano.

${ }^{3}$ Agencia de Protección Ambiental, (EPA 2002), agua de consumo humano.

${ }^{4}$ Organización de las Naciones Unidas para la Agricultura y la Alimentación (FAO, por sus siglas en inglés), Ayers (1976), calidad del agua para agricultura.

$\mathrm{T}=$ temperatura, $\mathrm{pH}=$ potencial de hidrógeno, $\mathrm{SDT}=$ sólidos disueltos totales, $\mathrm{TB}=$ turbidez, $\mathrm{CE}=$ conductividad eléctrica.

Por otra parte, se encontró que los valores de TB fueron variables tanto en muestras de río como en manantiales. Así, en las muestras de agua de río el intervalo de TB en unidades nefelométricas de turbidez (UNT) fue de 0.13 a 5.67 UNT, mientras que en agua de manantial el intervalo fue entre 0.03 y 17.8 UNT. Las muestras $5 \mathrm{R}$ y $1 \mathrm{~S}$ mostraron valores por encima del límite permisible para agua de consumo humano en la normatividad mexicana (SSA 1994). Es importante puntualizar que tomando en cuenta el valor del límite de calidad establecido por la Organización Mundial de la Salud (OMS) (0.1 UNT), la mayoría de las muestras se encuentran por arriba de dicho valor (WHO 2008). Sin embargo, en la muestra de agua del punto $6 \mathrm{~S}$ se obtuvo un valor de TB por debajo de los límites establecidos por todas las agencias aquí presentadas. La CE fue mayor en muestras de agua de río que en agua de manantial, donde los valores promedio fueron de 2.8 y $1.2 \mathrm{mS} / \mathrm{cm}$, respectivamente. Con ello, las muestras $1 \mathrm{R}, 2 \mathrm{R}$ y $3 \mathrm{~S}$ mostraron valores de $\mathrm{CE}$ por arriba de los límites establecidos para irrigación agrícola por la Organización de las Naciones Unidas para la Alimentación y la Agricultura (FAO) (Ayers 1976).

Además, en el presente estudio se analizó el contenido de varios elementos mayoritarios y traza. Los contenidos de As, $\mathrm{Be}, \mathrm{Cd}, \mathrm{Cr}, \mathrm{Pb}$ y $\mathrm{Sr}$ en las muestras colectadas se encontraron por debajo del límite de detección del equipo. Los resultados de las concentraciones de $\mathrm{Ca}, \mathrm{Mg}, \mathrm{Cu}, \mathrm{Li}, \mathrm{Ni}, \mathrm{Sb}, \mathrm{Se}$ y $\mathrm{Zn}$ se encuentran en el cuadro II.

La concentración promedio de Ca en agua de río fue más alta que en las muestras de agua de manantial, con valores de 8.7 y $6.2 \mathrm{mg} / \mathrm{L}$, respectivamente. Los contenidos de $\mathrm{Mg}$ fueron solamente detectados en muestras de agua de río en un intervalo de concentraciones de 0.7 a $1.9 \mathrm{mg} / \mathrm{L}$. De acuerdo con la normatividad, las concentraciones de estos elementos 
CUADRO II. CONCENTRACIÓN DE ELEMENTOS TRAZA (mg/L) EN MUESTRAS DE AGUA DE RÍO (BALLORECA, CONCHEÑO Y APITUCHI) Y MANANTIAL

\begin{tabular}{|c|c|c|c|c|c|c|c|c|c|}
\hline Muestra & $\mathrm{Ca}$ & $\mathrm{Mg}$ & $\mathrm{Li}$ & $\mathrm{Ni}$ & $\mathrm{Sb}$ & $\mathrm{Se}$ & $\mathrm{Cu}$ & $\mathrm{Zn}$ & $\begin{array}{c}\text { Dureza } \\
\left(\mathrm{mg} / \mathrm{L} \mathrm{CaCO}_{3}\right)^{*}\end{array}$ \\
\hline NORM $^{1}$ & & & & & & & 2 & 5 & 500 \\
\hline $\mathrm{WHO}^{2}$ & 75 & 50 & & 0.07 & 0.02 & 0.01 & 2 & 5 & 500 \\
\hline $\mathrm{EPA}^{3}$ & & & & & 0.006 & 0.05 & 1.3 & & \\
\hline $\mathrm{FAO}^{4}$ & 400 & 60 & & 0.2 & & & 0.2 & 2 & \\
\hline \multicolumn{10}{|l|}{ Río (R) } \\
\hline $1 \mathrm{R}$ & 10.9 & 0.7 & $<\mathrm{LD}^{\dagger}$ & $<\mathrm{LD}$ & $<\mathrm{LD}$ & $<\mathrm{LD}$ & 0.17 & $<\mathrm{LD}$ & 30.0 \\
\hline $2 \mathrm{R}$ & 4.5 & $<\mathrm{LD}$ & $<\mathrm{LD}$ & $<\mathrm{LD}$ & $<\mathrm{LD}$ & $<\mathrm{LD}$ & 0.18 & $<\mathrm{LD}$ & 11.4 \\
\hline $3 R$ & 14.0 & 1.9 & 0.04 & 0.84 & $<\mathrm{LD}$ & 0.003 & 0.43 & 0.14 & 42.6 \\
\hline $4 \mathrm{R}$ & 5.7 & $<\mathrm{LD}$ & 0.05 & 0.94 & $<\mathrm{LD}$ & 0.001 & 0.41 & 0.24 & 14.3 \\
\hline $5 \mathrm{R}$ & 6.5 & $<\mathrm{LD}$ & 0.20 & 0.84 & 0.015 & 0.048 & 0.57 & 0.27 & 16.2 \\
\hline $6 \mathrm{R}$ & 12.4 & 1.6 & 0.04 & 0.84 & $<\mathrm{LD}$ & $<\mathrm{LD}$ & 0.38 & 0.13 & 37.4 \\
\hline $7 \mathrm{R}$ & 6.7 & $<\mathrm{LD}$ & 0.04 & 0.80 & $<\mathrm{LD}$ & $<\mathrm{LD}$ & 0.35 & 0.08 & 16.8 \\
\hline \multicolumn{10}{|c|}{ Manantial (S) } \\
\hline $1 \mathrm{~S}$ & 9.00 & $<\mathrm{LD}$ & 0.04 & 0.76 & $<\mathrm{LD}$ & $<\mathrm{LD}$ & 0.36 & 0.06 & 22.5 \\
\hline $2 \mathrm{~S}$ & 7.90 & $<\mathrm{LD}$ & 0.03 & 0.86 & $<\mathrm{LD}$ & $<\mathrm{LD}$ & 0.41 & 0.14 & 19.7 \\
\hline $3 \mathrm{~S}$ & 6.49 & $<\mathrm{LD}$ & 0.04 & 0.88 & $<\mathrm{LD}$ & $<\mathrm{LD}$ & 0.43 & 0.13 & 16.2 \\
\hline $4 \mathrm{~S}$ & 1.99 & $<\mathrm{LD}$ & $<\mathrm{LD}$ & $<\mathrm{LD}$ & $<\mathrm{LD}$ & $<\mathrm{LD}$ & 0.16 & $<\mathrm{LD}$ & 5.0 \\
\hline $5 \mathrm{~S}$ & 6.31 & $<\mathrm{LD}$ & 0.03 & 0.86 & $<\mathrm{LD}$ & $<\mathrm{LD}$ & 0.43 & 0.13 & 15.7 \\
\hline $6 \mathrm{~S}$ & 6.49 & $<\mathrm{LD}$ & 0.20 & 0.84 & 0.015 & 0.048 & 0.57 & 0.27 & 16.2 \\
\hline $7 \mathrm{~S}$ & 5.72 & $<\mathrm{LD}$ & 0.04 & 0.77 & $<\mathrm{LD}$ & $<\mathrm{LD}$ & 0.36 & 0.06 & 14.3 \\
\hline $8 \mathrm{~S}$ & 6.08 & $<\mathrm{LD}$ & 0.05 & 0.84 & 0.004 & 0.009 & 0.46 & 0.18 & 15.2 \\
\hline
\end{tabular}

${ }^{1}$ NOM-127-SSA1-1994, (Secretaría de Salud 1994), agua de consumo humano.

${ }^{2}$ Organización Mundial de la Salud, (WHO 2008), agua de consumo humano.

${ }^{3}$ Agencia de Protección Ambiental, (EPA 2002), agua de consumo humano.

${ }^{4}$ Organización de las Naciones Unidas para la Agricultura y la Alimentación (FAO, por sus siglas en inglés), Ayers (1976), calidad del agua para agricultura.

*Calculados de las concentraciones de $\mathrm{Ca}$ y $\mathrm{Mg}$ en las muestras de agua

${ }^{\dagger}$ Limite de detección $=\mathrm{LD}_{\text {equipo }}<1 \mu \mathrm{g} / \mathrm{L}$

son menores a los límites permisibles. Asimismo, la dureza fue calculada utilizando los contenidos de $\mathrm{Ca}$ y $\mathrm{Mg}$. El intervalo determinado fue de 11.4 a $42.6 \mathrm{mg} / \mathrm{L} \mathrm{CaCO}_{3}$ y de 5 a $22.5 \mathrm{mg} / \mathrm{L} \mathrm{CaCO}_{3}$, en las muestras de agua colectadas en río y manantial, respectivamente.

\section{DISCUSIÓN}

Los valores de los parámetros fisicoquímicos obtenidos en las muestras de agua fueron bajos, excepto para la TB. La principal causa de la TB en el agua es el material particulado debido a la resuspensión de los sedimentos. El valor más alto de TB en agua de río se encontró en la muestra 5R. Asimismo, en el agua de manantial de la muestra $1 \mathrm{~S}$ se obtuvo un valor de 17.8 UNT. Es importante mencionar que el agua de este manantial se utiliza principalmente para consumo humano en esa región. En este punto de muestreo, el agua proviene de una mezcla de flujos de dos manantiales lo cual causa resuspensión de los sedimentos del fondo en el punto de muestreo. Además, adyacentes a este punto muestreado existen jales de la minería independiente (gambusinos) los cuales, por procesos de erosión y lixiviación, contribuyen con iones disueltos y partículas sedimentables. Para el agua de consumo humano un valor de TB de 5 UNT es por lo general aceptable (SSA 1994), aunque esto puede variar de acuerdo con las características de la fuente. Sin embargo, los valores de TB recomendados deben estar por debajo de 0.1 UNT para la calidad del agua de consumo humano según la OMS (WHO 2008). Ningún efecto sobre la salud ha sido propuesto por un valor alto de TB. Sin embargo, es de gran importancia en la calidad de agua para condiciones de vida acuática (CCME 2007). Asimismo, la CE mostró valores altos en las muestras $1 \mathrm{R}, 2 \mathrm{R}$ y $3 \mathrm{~S}$. Todas ellas fueron tomadas en la cuenca del río Apituchi, lo cual concuerda con 
las altas concentraciones de SDT en esos puntos de muestreo. Además, los valores calculados de dureza son menores a los límites considerados en la regulación nacional e internacional, dichos valores indican que el agua de los ríos y los manantiales aquí analizados se puede catalogar como "agua blanda" $\left(<50 \mathrm{mg} / \mathrm{L} \mathrm{CaCO}_{3}\right)$.

Los elementos traza medidos en las muestras de agua se encontraron en la tendencia siguiente: $\mathrm{Ni}>$ $\mathrm{Cu}>\mathrm{Zn}>\mathrm{Li}>\mathrm{Se}$. La mayoría de los elementos traza fueron menores en comparación con los límites establecidos para agua de consumo humano presentados por la Agencia de Protección Ambiental de Estados Unidos de América (EPA 2000) y la normatividad mexicana (SSA 1994). Por el contrario, el Sb sí se presentó en algunas muestras en concentraciones mayores al límite permisible. Este elemento fue detectado sólo en algunas muestras, con un intervalo de concentraciones entre 0.004 y $0.015 \mathrm{mg} / \mathrm{L}$. Además, en dos muestras de agua de río ( $3 \mathrm{R}$ y $4 \mathrm{R}$ ) las concentraciones de Se estuvieron por encima de los límites marcados por la EPA.

De manera general, el agua continental presenta concentración baja de Ni (Ayers 1976, WHO 2008). El Ni no es tomado en cuenta por la normatividad para agua de consumo en la EPA y en la regulación mexicana. Sin embargo, las concentraciones encontradas en todas las muestras son mayores a las establecidas por la OMS. Las muestras de agua mostraron concentraciones altas de Ni tanto para ser usadas como agua de consumo o para la agricultura. Se sugiere que las concentraciones altas de Ni son debidas a las características geológicas de la región, aunque en el presente trabajo no se realizó la caracterización elemental del material rocoso.

Por otra parte, en la zona de estudio se localiza mineralización de elementos tales como $\mathrm{Au}, \mathrm{Ag}, \mathrm{Cd}$, $\mathrm{Cu}, \mathrm{Pb}, \mathrm{Sb}, \mathrm{Zn}$ y $\mathrm{Hg}$ (SGM 2010). En esta misma zona se encuentran reportados los contenidos de $\mathrm{Cu}$, $\mathrm{Sb}$ y $\mathrm{Zn}$ en sedimentos de arroyos. Así, las concentraciones de $\mathrm{Cu}$ en sedimentos de algunos arroyos de la zona se encuentran en un intervalo de 0.9 a 22 ppm (SGM 2000a), mientras que en las muestras de agua del presente estudio fueron de 0.17 a $0.57 \mathrm{mg} / \mathrm{L}$. Tales concentraciones se encuentran por debajo de los límites permisibles para el agua de consumo. No obstante, en la mayoría de las muestras dichas concentraciones se encuentran por encima del límite permitido por la FAO para el agua de riego (Cuadro II). Aunque en esta región no se practica ampliamente la agricultura, hay pequeñas granjas donde se tiene, a menor escala, la práctica de cultivo para el consumo familiar. De igual manera, el Servicio Geológico
Mexicano reporta que en sedimentos de arroyo localizados en los alrededores del Pilar, las concentraciones de Sb fueron hasta de 396 ppm (SGM 2000b). En esa comunidad se colectó agua de manantial (punto $1 \mathrm{~S}$ ), donde la concentración de este elemento no fue detectada. De igual manera, los sedimentos de los arroyos presentes en el resto de la zona de estudio mostraron concentraciones de $\mathrm{Sb}$ de 2.2 a $4.7 \mathrm{ppm}$ (SGM 2000b). Por otra parte, es conocido que el Se frecuentemente se encuentra en asociación con minerales que contienen azufre (ATSDR 2003) mientras que Sb se asocia a óxidos (ATSDR 1992). En el presente estudio, el agua obtenida en los puntos $5 \mathrm{R}$ y 6S mostraron concentraciones altas de $\mathrm{Cu}$, Se y $\mathrm{Sb}$ en comparación con el resto de los puntos (Cuadro II). Por lo anterior, ambos elementos presentes tanto en las muestras de agua de manantial como en las de río, pueden estar siendo transportados principalmente por sedimentos y el material particulado suspendido en agua. Estas muestras de agua fueron tomadas en una zona altamente alterada por las actividades antrópicas relacionadas con la actividad minera, específicamente por el cambio de uso de suelo (incendios forestales, bancos de materiales, jales mineros, caminos, cultivos, entre otros).

Por otra parte, las concentraciones de $\mathrm{Zn}$ en las muestras de agua estuvieron por debajo de los límites permisibles establecidos por las diferentes instituciones tomadas en cuenta en este trabajo. Además, aunque el valor límite permisible de $\mathrm{Zn}$ se encuentre en $5 \mathrm{mg} / \mathrm{L}$, la OMS recomienda que el agua que contenga $3 \mathrm{mg} / \mathrm{L}$ no es apta para consumo humano (WHO 2008). Algunos autores han reportado valores que han alcanzado concentraciones $>500 \mathrm{mg} / \mathrm{L}$ de $\mathrm{Zn}$ en agua superficial en estudios realizados en zonas circundantes a un distrito minero (Ramos et al. 2014). Valores de concentración que son muy altos en comparación con lo encontrado en nuestro estudio (valor máximo de concentración de $0.27 \mathrm{mg} / \mathrm{L}$; Cuadro II). La concentración del Zn en el agua está en función del $\mathrm{pH}$, potencial de reducción oxidación (Eh) y de otras especies químicas presentes (SRHNA 2004, WHO 2008). En agua superficial, el Zn comúnmente se encuentra con valencia +2 en especies químicas tales como ion libre $\left(\mathrm{Zn}^{+2}\right)$ y compuestos inorgánicos como $\mathrm{ZnSO}_{4}$ y $\mathrm{ZnCO}_{3}$. Como $\mathrm{ZnSO}_{4}$, es soluble en agua y provoca un sabor astringente indeseable al agua a una concentración mayor de $4 \mathrm{mg} / \mathrm{L}$. Como carbonato de zinc su solubilidad disminuye con el aumento de $\mathrm{pH}$ (WHO 2008). En la figura 2 se muestra la correlación encontrada entre las concentraciones de $\mathrm{Zn}$ respecto al $\mathrm{pH}$. Las muestras de agua de los ríos mostraron $\mathrm{pH}$ en un intervalo de neutro a ligeramente 

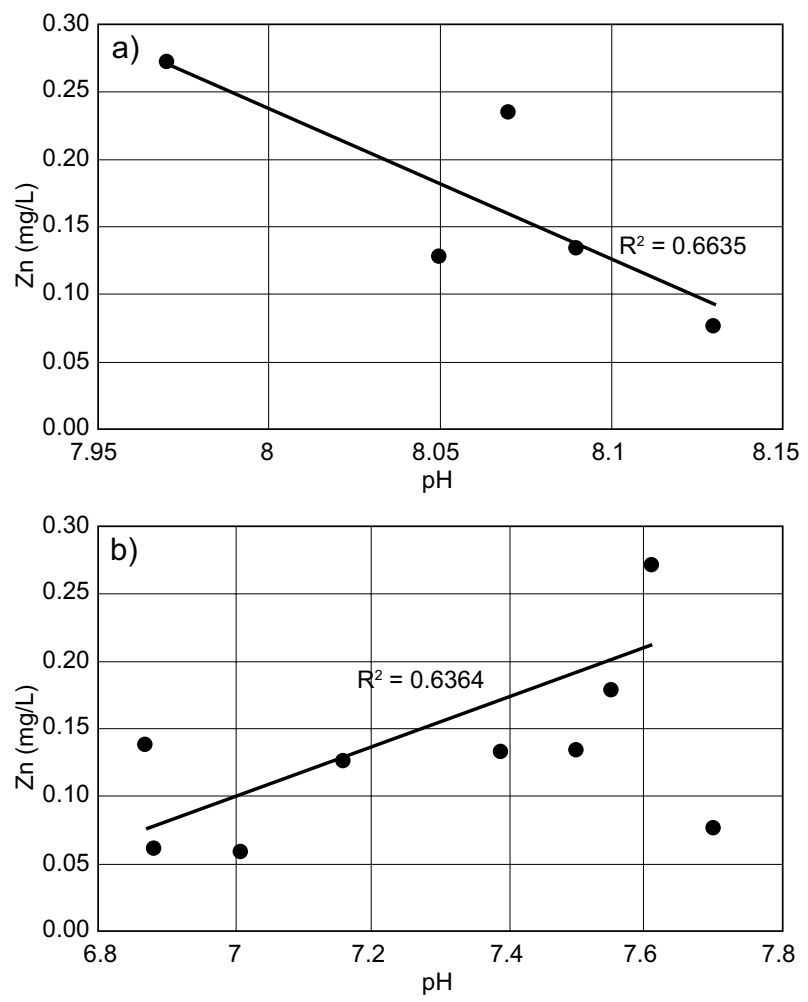

Fig. 2. Correlación lineal de pH con las concentraciones de $\mathrm{Zn}$ en las muestras de agua de: a) río y b) manantial

básico, donde la concentración de Zn manifestó una tendencia a disminuir $\left(\mathrm{R}^{2}=0.66\right.$ y $\left.\mathrm{p}=0.0002\right)$ al decrecer el valor del $\mathrm{pH}$ (Fig. 2a). Por el contrario, en muestras de agua tomadas en manantiales, donde los valores de $\mathrm{pH}$ oscilaron desde ligeramente ácido a neutro, se observó que la concentración de $\mathrm{Zn}$ se incrementa $\left(\mathrm{R}^{2}=0.64 \mathrm{y} \mathrm{p}=0.1\right)$ con el aumento del pH (Fig. 2b). En agua con condiciones de oxidación y $\mathrm{pH}$ cercanos a 7, el $\mathrm{Zn}$ es principalmente encontrado como ion libre y con bajas concentraciones como hidróxido o carbonato (SRHNA 2004). Con lo anterior, podemos sugerir que en agua de río de nuestra zona de estudio el $\mathrm{Zn}$ se encuentra asociado a carbonatos en el intervalo de $\mathrm{pH}$ medido. Por ello, el $\mathrm{ZnCO}_{3}$ tenderá a precipitarse sobre superficies de sedimentos debido a su baja solubilidad $(0.21 \mathrm{~g} / \mathrm{L})$. Lo anterior corrobora las concentraciones de $\mathrm{Zn}$ en sedimentos de arroyos en la zona de estudio, con intervalo de concentraciones de 17.3 a 83 ppm (SGM 2000c). Además del pH, se observó que existe una correlación lineal de los contenidos de $\mathrm{Zn}$ y $\mathrm{Cu}$, para el agua de río $\left(\mathrm{R}^{2}=0.67\right)$ y de manantial $\left(\mathrm{R}^{2}=0.77\right)$. Como resultado, el $\mathrm{Zn}$ y Cu presentarán sus máximas concentraciones en agua a $\mathrm{pH}$ alrededor de 7.98. Por lo anterior, se puede sugerir que a un mayor $\mathrm{pH}$ a partir de 7, estos elementos probablemente se encuentren adsorbidos a los sedimentos.

En este estudio se calcularon los elementos traza en muestras de agua. Sin embargo, la mayoría de los elementos tienen que ser evaluados en los sedimentos debido a su alta reactividad, así como a los fenómenos de adsorción, desorción, precipitación y resuspensión, para así obtener una mejor comprensión del fenómeno de interacción sólido/solución.

Tanto la evaluación de los parámetros físicos y químicos como la caracterización de los elementos pueden indicar una adecuada calidad de agua para el desarrollo de la vida acuática. En consecuencia, algunos países tienen normas bien establecidas para la protección de la vida acuática. Por ejemplo, el Consejo Canadiense de Medio Ambiente toma en cuenta los valores de $0.004,0.025$ y $0.05 \mathrm{mg} / \mathrm{L}$, como límites permisibles para $\mathrm{Cu}$, Ni y Zn, respectivamente (CCME 2007). Sin embargo, la EPA considera un valor más estricto permisible de concentración de Zn en agua de $0.012 \mathrm{ppm}$ (EPA 2002) para no afectar la vida acuática. En comparación con estas regulaciones, las muestras de agua de río analizadas presentaron valores más altos de concentraciones de $\mathrm{Cu}, \mathrm{Ni}$ y $\mathrm{Zn}$. Por lo tanto, en las zonas donde las actividades antrópicas están involucradas deben vigilarse las concentraciones de elementos traza. Además, otros autores han mencionado que valores altos de TB en cuerpos de agua podrían afectar tanto en cantidad como en calidad la producción de pescado (Singler, et al. 1984). Es recomendable evaluar y monitorear los contenidos de elementos traza en rocas, suelos, sedimentos de los ríos, flora y fauna del distrito minero de Ocampo, con el fin de tener una mejor comprensión de la movilidad de tales elementos y su posible influencia como contaminantes.

\section{CONCLUSIONES}

Las actividades mineras están frecuentemente relacionadas con los impactos ambientales, incluyendo la contaminación por metales pesados en los sistemas acuáticos. Aunque, las concentraciones de elementos determinadas en este trabajo son menores que los límites permitidos por la EPA y por la regulación mexicana para agua de consumo humano, algunos de ellos superan los permisibles por la OMS. Lo anterior es debido en gran medida a las características geológicas de la región. Sin embargo, se determinó que el río Balloreca está siendo impactado por la actividad antrópica en la zona cercana a los jales mineros debido a la presencia 
de elementos tales como $\mathrm{Cu}, \mathrm{Li}, \mathrm{Sb}, \mathrm{Se}$ y $\mathrm{Zn}$. Se concluye también, que en los cuerpos de agua, el $\mathrm{pH}$ juega un papel importante en la presencia de especies químicas como son el Cu, el Ni y el Zn. Sin embargo, por diversos estudios se observa que son los sedimentos quienes son una fuente importante de transporte de metales pesados en la región estudiada. Debido a que el agua tiene TB alta, se plantea que las especies químicas cuantificadas se encuentran asociadas al material particulado suspendido. La realización de este tipo de estudios es importante debido a que representa una línea base de análisis que permite plantear estrategias para reducir los efectos negativos que afectan el desarrollo de la biota, principalmente en cuerpos de agua. Asimismo, se sugiere establecer medidas de remoción de elementos tóxicos en el agua, tanto de manantiales como de ríos, cuando el agua de dichas fuentes sea utilizada para consumo humano con la intención de disminuir cualquier efecto adverso a la salud de la población.

\section{AGRADECIMIENTOS}

Los autores agradecen el apoyo brindado por el Consejo Nacional de Ciencia y Tecnología (CONACyT) dentro del proyecto CB-2011- 16697, así como al Ing. Rafael Escobedo por su contribución.

\section{REFERENCIAS}

Alloway B.J. y Ayres D.C. (1993). Chemical principles of environmental pollution. Blackie Academic and Professional. Nueva York, EUA, 291 pp.

Ansari A. A., Singh I. B. y Tobschall H. J. (1999). Status of anthropogenically induced metal pollution in the Kanpur Unnao industrial region of the Ganga plain, India. Environm. Geo. 38, 25-33.

DOI: $10.1007 / \mathrm{s} 002540050397$

ATSDR (1992). Toxicological profile for Antimony. Agency for Toxic Substances and Disease Registry. Federal public health agency of the U.S. Department of Health and Human Services, 7440-36-0 [en línea]. http://www.atsdr.cdc.gov/ToxProfiles/ tp.asp?id=332\&tid=58\#bookmark15 10/12/2015.

ATSDR (2003). Toxicological profile for Selenium. Agency for Toxic Substances and Disease Registry. Federal public health agency of the U.S. Department of Health and Human Services, 7782-49-2 [en línea]. http://www. atsdr.cdc.gov/ToxProfiles $/$ TP.asp id $=153 \&$ tid $=28$ $10 / 12 / 2015$.
Ayers R.S. y Westcot D.W. (1976). Water quality for agriculture. FAO irrigation and drainage paper 29, 1-96 pp.

Azarcoya González B. A. (2012). La Sierra Tarahumara, el bosque y los pueblos originarios: estudio de caso de Chihuahua México [en línea]. http://www.fao.org/forestry/17194-0381f923a6bc236aa91ecf614d92e12e0.pdf 30/11/2014.

Bridge G. (2004). Contested terrain: Mining and the environment. Annu. Rev. Environ. Resour. 29, 205-259. DOI: 10.1146/annurev.energy.28.011503.163434

Bud I., Duma S., Denuţ I. y Taşcu I. (2007). Water pollution due to mining activity. Causes and consequences. BHM Berg-Und Hüttenmännische Monatshefte 152, 326. DOI: $10.1007 / \mathrm{s} 00501-007-0321-x$

CCME (2007). A protocol for the derivation of water quality guidelines for the protection of aquatic life 2007. Canadian Council of Ministers of the Environment [en línea]. http://www.ccme.ca/files/Resources/ supporting_scientific_documents/protocol_aql_2007e. pdf 30/08/2015.

Carrillo-Chávez A., Morton-Bermea O., González-Partida E., Rivas-Solorzano H., Oesler G., García-Meza V. y Cienfuegos E. (2003). Environmental geochemistry of the Guanajuato mining district, Mexico. Ore. Geol. Rev. 23, 277-297.

DOI: $10.1016 / \mathrm{S} 0169-1368(03) 00039-8$

CONANP (2014). Comisión Nacional de Áreas Naturales Protegidas. Programa de Manejo Área de Protección de Flora y Fauna Tutuaca. Primera Edición [en línea]. http://www.conanp.gob.mx/que_hacemos/pdf/programas_manejo/2014/Tutuacafinal.pdf 15/12/2015.

EPA (2000). National primary drinking water regulations: Ground water rule (40 CFR Parts 141 and 142). Environmental Protection Agency, EUA, 100 pp.

EPA (2002). National recommended water quality criteria. Environmental Protection Agency [en línea]. http:// training.fws.gov/resources/course-resources/pesticides/General/WQC-2002.pdf.

EPA (2007). Microwave assisted acid digestion of aqueous samples and extracts. Método 3015A. Environmental Protection Agency [en línea]. http://www3.epa.gov/ epawaste/hazard/testmethods/sw846/pdfs/3015a.pdf 10/06/2015.

Fernández-Manso A., Quintano C. y Roberts D. (2012). Evaluation of potential of multiple endmember spectral mixture analysis (MESMA) for surface coal mining affected area mapping in different world forest ecosystems. Remote Sens. Environ. 127, 181-193. DOI: 10.1016/j.rse.2012.08.028

Gómez-Álvarez A., Valenzuela-García J. L., MezaFigueroa D., de la O Villanueva M., Ramírez-Hernández J., Almendariz-Tapia J. y Pérez-Segura E. (2011). Impact of mining activities on sediments in a semi-arid 
environment: San Pedro River, Sonora, Mexico. Appl. Geochem. 26, 2101-2112.

DOI: 10.1016/j.apgeochem.2011.07.008

Hilson G. y Van Der Vorst R. (2002). Technology, managerial, and policy initiatives for improving environmental performance in small-scale gold mining industry. Environ. Manage. 30, 764-777.

DOI: $10.1007 / \mathrm{s} 00267-002-2728-1$

Hun-Bok J., Seong-Tack Y., Bernhard M., Soon-Oh K., Seong-Sook P. y Pyeong-Koo L. (2005). Transport and sediment-water partitioning of trace metals in acid mine drainage: an example from the abandoned Kwangyang $\mathrm{Au}-\mathrm{Ag}$ mine area, South Korea. Environ. Geol. 48, 437-449.

DOI: $10.1007 / \mathrm{s} 00254-005-1257-7$

INEGI (2005a). Prontuario de información geográfica municipal de los Estados Unidos Mexicanos. Moris, Chihuahua, clave geoestadística 08047. Instituto Nacional de Estadística y Geografía. [en línea]. http:// www3.inegi.org. $\mathrm{mx} / \mathrm{sistemas} / \mathrm{mexicocifras} /$ datosgeograficos/08/08047.pdf 02/02/2015.

INEGI (2005b). Prontuario de información geográfica municipal de los Estados Unidos Mexicanos. Ocampo, Chihuahua, clave geoestadística 08051. Instituto Nacional de Estadística y Geografía. [en línea]. http:// www3.inegi.org.mx/sistemas/mexicocifras/datosgeograficos/08/08051.pdf 02/02/2015.

Kumah A. (2006). Sustainability and gold mining in the developing world. J. Clean. Prod. 14, 315-323.

DOI: 10.1016/j.jclepro.2004.08.007

Metcalf K. y Eddy I. (1996). Ingeniería de Aguas Residuales. Description and model representation. McGraw Hill. Wat. SA 14, 185-192 pp.

Ramos R. O. E., Rötting T. S., French M., Sracek O., Bundschuh J., Quintanilla J. y Bhattacharya P. (2014). Geochemical processes controlling mobilization of arsenic and trace elements in shallow aquifers and surface waters in the Antequera and Poopó mining regions, Bolivian Altiplano. J. Hydrol. 518, 421-433. DOI: 10.1016/j.jhydrol.2014.08.019

SEMARNAT (1996). Norma Oficial Mexicana NOM001-ECOL-1996. Límites máximos permisibles de contaminantes en las descargas de aguas residuales en aguas y bienes nacionales. Secretaría de Medio Ambiente y Recursos Naturales. Diario Oficial de la Federación. 24 de junio de 1996.

SGM (2000a). Carta geoquímica por $\mathrm{Cu}$, Tecoripa H1212, Sonora y Chihuahua. Levantamiento geoquímico por sedimentos de arroyo. Informe Final. Servicio Geológico Mexicano. [en línea]. http://mapserver.sgm. gob.mx/Cartas_Online/geoquimica/28_H12-12_cu.pdf $15 / 02 / 2015$.
SGM (2000b). Carta geoquímica por Sb, Tecoripa H12-12, Sonora y Chihuahua. Informe Final. Servicio Geológico Mexicano. [en línea]. http://mapserver.sgm. gob.mx/Cartas_Online/geoquimica/28_H12-12_sb.pdf $15 / 02 / 2015$.

SGM (2000c). Carta geoquímica por Zn, Tecoripa H12-12, Sonora y Chihuahua. Servicio Geológico Mexicano [en línea]. http://mapserver.sgm.gob.mx/Cartas_Online/ geoquimica/28_H12-12_zn.pdf 16/02/2015.

SGM (2010). Carta geológico-minera. Servicio Geológico Mexicano [en línea]. http://mapserver.sgm. gob.mx/Cartas_Online/geologia/28_H12-12_GM.pdf 11/09/2015.

Sigler J. W., Bjornn T. C. y Everest F. H. (1984). Effects of chronic turbidity on density and growth of steelheads and coho salmon. Trans. Am. Fish Soc. 113, 142-150. DOI: $10.1577 / 1548-8659(1984) 113<142$ :EOCTOD $>2.0 . \mathrm{CO} ; 2$

SRHNA (2004). Desarrollos de niveles guía nacionales de calidad de agua ambiente correspondientes a zinc. Subsecretaría de Recursos Hídricos de la Nación, Argentina [en línea]. http://www.produccion-animal. com.ar/agua_bebida/26-zinc.pdf 02/01/2016.

Srinivasa Gowd S. y Govil P. K. (2008). Distribution of heavy metals in surface water of Ranipet industrial area in Tamil Nadu, India. Environ. Monit. Assess. 136, 197-207. DOI: $10.1007 / \mathrm{s} 10661-007-9675-5$

SSA (1994). Modificación a la norma NOM-127SSA1-1994, Salud Ambiental. Agua para uso y consumo humano. Límites permisibles de calidad y tratamientos a que debe someterse el agua para su potabilización. Secretaría de Salud Ambiental. Diario Oficial de la Federación. 22 de noviembre de 2000.

Torres P., Cruz C. H. y Patiño P. J. (2009). Índices de calidad de agua en fuentes superficiales utilizadas en la producción de agua para consumo humano. Una revisión crítica. Revista Ingenierías Universidad de Medellín. 8, 79-94.

Villanueva J., Fulé P., Paredes C., Estrada J. y Sánchez I. (2009). Reconstrucción de la precipitación estacional para el barlovento de la Sierra Madre Occidental con anillos de crecimiento de Pseudotsuga menziesii (Mirb.) Franco. Revista Ciencia Forestal en México 34, 37-69.

WHO (2008). Guidelines for drinking-water quality. 3ra. ed. World Health Organization, Ginebra, Suiza, 492 pp.

Younger P. L. (1997). The longevity of minewater pollution: A basis for decision-making. Sci. Total Environ. 194, 457-466.

DOI: $10.1016 / \mathrm{S} 0048-9697(96) 05383-1$ 